\title{
Prevalencia de malaria en aves de sotobosque en la cuenca alta del rio Itaya, Loreto, Perú
}

Paula A. Galvez Zagaceta ${ }^{1,2}$, Tino A. Vela Trigoso', Segundo L. Estela Moreno¹, Hicler N. Rodriguez Mashacuri ${ }^{1,2}$, Juan C. Castro Gómez ${ }^{2}$, Marianela Cobos Ruiz ${ }^{1,2}$, Jae D. Paredes Rodríguez ${ }^{1,2}$, Joseph Dylan Maddox $1,3,4$

${ }^{1}$ Laboratorio de Biotecnología y Bioenergética (LBB) - Universidad Científica del Perú (UCP), Av. José Abelardo Quiñones Km 2.5, San Juan Bautista, lquitos, Perú

2Unidad Especializada del Laboratorio de Investigación de Biotecnología (UELIB), Centro de Investigación de Recursos Naturales de la UNAP (CIRNA)-Universidad Nacional de la Amazonia Peruana (UNAP), Psje. Los Paujiles S/N, San Juan Bautista, Iquitos, Perú

${ }^{3}$ Pritzker Laboratory for Molecular Systematics and Evolution, Field Museum of Natural History, 1400 S. Lake Shore Drive, Chicago, IL 60605, USA

${ }^{4}$ Environmental Sciences, American Public University System, Charles Town, WV 25414, USA

Enviado el 15 de junio del 2021. Aceptado el 28 de junio 2021.

DOI: https://doi.org/10.33017/RevECIPeru2021.0004/

\section{Resumen}

La Amazonía peruana y en particular la región Loreto, alberga una gran diversidad de aves que han sido estudiadas en diferentes campos. Sin embargo, se conoce muy poco acerca de sus enfermedades. Una de las enfermedades de mayor frecuencia en aves es la malaria aviar, causada por los hemosporidios. No obstante, existe una carencia de información sobre la prevalencia de malaria en esta zona, y de la interacción huéspedparásito, los daños tisulares, la supervivencia y otros efectos que pueden causados por estos parásitos. El objetivo de esta investigación fue determinar la prevalencia de malaria en aves de sotobosque de la cuenca alta rio Itaya. Se obtuvieron muestras de sangre de 281 aves y se detectó malaria aviar mediante métodos moleculares. Se identificaron 66 especies de aves pertenecientes a 18 familias. Asimismo, se ha registrado una prevalencia global de malaria aviar de 60,1\%, siendo la familia Thamnophilidae que mostró la mayor prevalencia de hemoparásitos (64,9\%). En conclusión, la prevalencia de malaria en aves de sotobosque de la cuenca alta del rio Itaya es alta, pudiendo representar un riesgo para la conservación de la diversidad de la avifauna en la Amazonía peruana.

Descriptores: malaria aviar, hemosporidios, prevalencia, métodos moleculares.

\section{Abstract}

The Peruvian Amazon and specifically the Loreto region harbours a great diversity of birds that have been studied in different fields. However, little is known about the diseases they afflict them. One type of disease disease is the avian malaria caused by hemosporidia. There is a gap of information regarding this and other diseases and valuable information such as host-parasite interactions, tissue damage, survival and other effects that can cause these parasites. The objective of this study was to determine the prevalence of malaria in understory birds of the upper Itaya river basin. Blood samples were obtained from 281 birds and the avian malaria parasites genetic material was detected by molecular methods. A total of 66 bird species belonging to 18 families were identified. An overall prevalence of avian malaria of $60.1 \%$ was recorded, with the Thamnophilidae family showing the highest prevalence of hemoparasites (64.9\%). In conclusion, the prevalence of malaria in understory birds in the upper Itaya river basin is high and could represent a risk for the conservation of avifaunal diversity in the Peruvian Amazon.

Keywords: avian malaria, hemosporidia, prevalence, molecular methods. 


\section{Introducción}

La Amazonía peruana y la región Loreto en particular, alberga una gran diversidad de aves, que han sido estudiadas a nivel de distribución, alimentación y ecología, pero muy poco se conoce acerca des las enfermedades que estas sufren. Un mejor conocimiento de las enfermedades que afectan a estas aves, podrian explicar la alta frecuencia de mortalidad que enfrentan las aves [1]. Dentro de este grupo de enfermedades, se encuentra la malaria aviar. Esta enfermedad es causada por un grupo diverso de parásitos protozoarios[2], estos son transmitidos por vectores dípteros hematófagos, que ejercen efectos patógenos en el ave provocando daño tisular [3],

\section{Metodología}

\subsection{Captura e identificación de aves}

Las aves fueron capturadas en el área de Concesión de Conservación Cuenca Alta Río Itaya (CCCARI) de la Universidad Científica del Perú UCP ubicada entre los ríos Amazonas, Marañón y Nanay al sureste de la ciudad de lquitos en el distrito

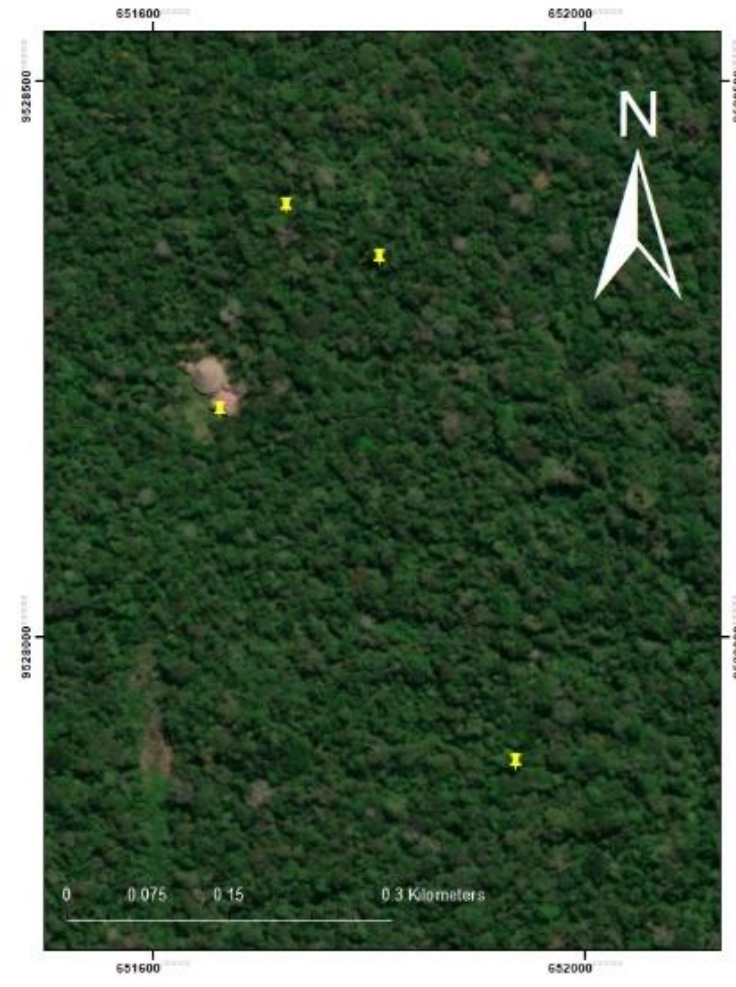

reduciendo su supervivencia y capacidad reproductiva [4]

Además, de causar daños en la reproducción de las aves, una de las características más interesantes de los hemosporidianos es su capacidad de infectar una amplia variedad de especies de aves [5], [6]. Varias investigaciones indican que estos patógenos están presentes en aves de casi todas las regiones biogeográficas del mundo, sin embargo los estudios en el neotrópico son fragmentarios y escasos [7]. Similarmente, en la Amazonía peruana y en la región Loreto los estudios sobre malaria aviar son limitados. Por tanto, el objetivo de esta investigación fue determinar la prevalencia de malaria en aves de sotobosque de la cuenca alta rio Itaya.

de San Juan Bautista, provincia de Maynas y departamento de Loreto [8] (Figura 1 ); utilizando redes de neblina colocadas a, nivel de sotobosque de forma individual o en sistemas de 2 o 3 redes, según las condiciones del terreno; estas se extrajeron manualmente evitando estresarlos e inmediatamente fueron colocados en bolsas de tela para ser trasladados al punto de procesamiento [9].

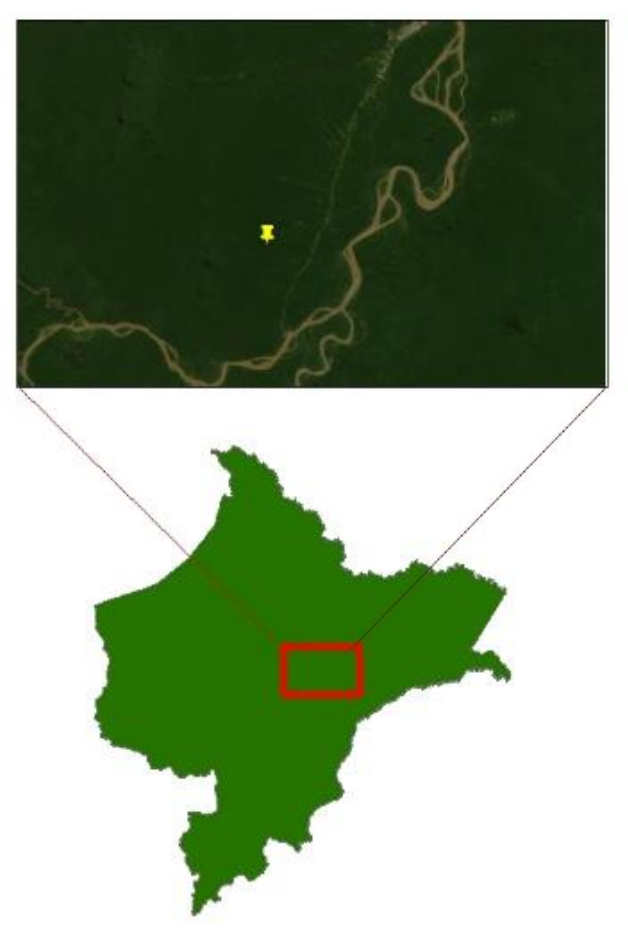

Figura 1: Área de muestreo en la Concesión de Conservación Cuenca Alta Río Itaya (CCCARI) de la Universidad Científica del Perú - UCP

\subsection{Marcación y morfometría de aves}

Las aves capturadas fueron identificadas morfológicamente según Schulenberg et al. [10] y a nivel de especie según Plenge [11], así mismo se 
tomaron datos morfométricos básicos, se registraron fotográficamente y se marcaron para evitar duplicidad en los registros.

\subsection{Toma y conservación de muestras de sangre.}

Se obtuvo mediante punción de la vena braquial del ala [12]. La sangre fue colectada en capilares heparinizados para luego ser transferidos a tarjetas Whatman FTA (GE Heathcare, EE. UU.), se codificó y almacenó a temperatura ambiente dentro de un envase con gel de sílice, hasta la realización de las pruebas moleculares.

\subsection{Detección molecular de malaria aviar}

Se utilizó el protocolo de precipitación por sales (Salting-out) modificado [13], que consistió en extraer una pequeña parte de sangre impregnada en las tarjetas Whatman FTA, colocándolos en microtubos para adicionar $200 \mu$ de TNES a pH 8 y $5 \mu \mathrm{l}$ de proteinasa $\mathrm{K}(10 \mathrm{mg} / \mathrm{ml})$, llevándolo a incubar a $56^{\circ} \mathrm{C}$ por 5 horas, se agregó $85 \mu \mathrm{l}$ de Cloruro de sodio $(\mathrm{NaCl}) 5 \mathrm{M}$ y se homogenizó por vortex (ISOLAB, modelo MX-S) a máxima revolución por 15 segundos. Se centrifugó (Thermoscientific, modelo MicroCL21R ) a $21000 x g$ por 7 minutos, se decantó el sobrenadante en un nuevo microtubo de $1,5 \mathrm{ml}$, agregando asimismo $500 \mu \mathrm{l}$ de isopropanol al $95 \%$, se volvió a centrifugar por $15 \mathrm{~min}$ y con la ayuda de una micropipeta, se descartó el isopropanol, agregando $500 \mu \mathrm{l}$ de Etanol frio al $70 \%$, volviendo a centrifugar por 10 minutos, finalmente se descartó el sobrenadante para luego secarlo a $55^{\circ} \mathrm{C}$ de $10-15$ min en un termoblock (USA SCIENTIFIC, INC, modelo P.O.Box 3565) y finalmente se agregó $100 \mu \mathrm{l}$ de buffer IDTE.

Luego se procedió a amplificar mediante la reacción en cadena de la polimerasa en tiempo real de acuerdo a Bell et al. [14] empleando los cebadores R330F (5'-CGTTCTTAACCCAGCTCACG-3') y R480RL (5'-GCCTGGAGGTWAYGTCC-3'), amplificados bajo las siguientes condiciones en un termociclador: $95^{\circ} \mathrm{C}$ para30 s, seguido de 35 ciclos de $95^{\circ} \mathrm{C}$ durante $30 \mathrm{~s}$ y $53^{\circ} \mathrm{C}$ durante $35 \mathrm{~s}$ (con una lectura de placa) seguido de una curva de fusión final, utilizando la configuración predeterminada del instrumento. Se incluyeron controles negativos en todas las reacciones.

\subsection{Análisis y Procesamiento de Datos}

Para calcular la prevalencia se utilizó la formula citada por Bell, J.R.[15], los datos adquiridos fueron ordenados y analizados en Excel 2010.

\section{Resultados y Discusión de Resultados}

\subsection{Identificación de las aves}

De un total de 281 aves capturadas se identificaron que pertenecen a 66 especies y 18 familias tales como: Thamnophilidae, furnariidae, pipridae, tyrannidae, troglodytidae, ramphastidae, thraupidae, onycorhynchidae, formicariidae, columbidae, cardinalidae, bucconidae, alcedinidae, galbulidae, turdidae, Capitonidae y Grallariidae. (Figura 2).

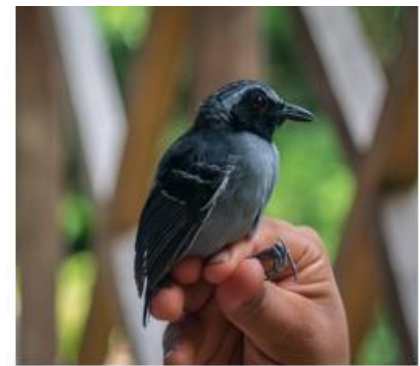

Myrmoborus myotherinus

(Thamnophilidae)

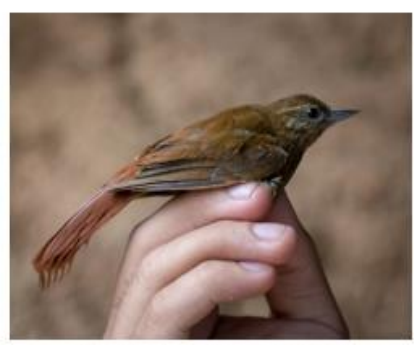

Glyphorynchus spirurus

(Furnariidae)

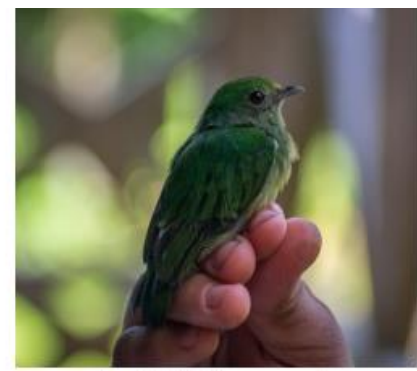

Lepidothrix coronata

(Pipridae)

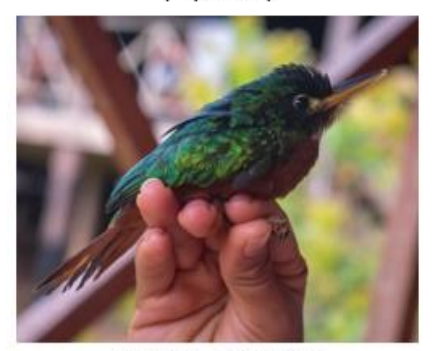

Galbula albirostris

(Galbulidae)
Figura 2: Algunas especies de aves de sotobosque de la Cuenca Alta Río Itaya capturadas durante el estudio

\subsection{Prevalencia de malaria aviar}

Los resultados obtenidos mediante PCR en tiempo real (Figura 2), nos muestra que, de los 281 ejemplares analizados, 169 individuos resultaron positivos con malaria aviar, representando una prevalencia global de $60,1 \%$ para la población estudiada. En contraste, un estudio sobre malaria aviar en la Reserva Nacional Allpahuayo Mishana reportó una prevalencia de $16,25 \%$ en un total de 240 ejemplares analizados [16].

Asimismo, en nuestro estudio se pudo observar que solo una familia no presentó infección (Grallariidae), 
en contraposición, la familia con más individuos positivos para malaria aviar fue Tamnophilidae $(n=50)$ lo cual indica una prevalencia de $64,9 \%$, seguido de la familia Pipridae $(63,2 \%)$ y la familia Furnariidae (49,1\%) (Figura 3). Sin embargo, el estudio de prevalencia realizado por Ricopa \& Villa [16] indica que la especie que presentó mayor prevalencia de malaria aviar fue Cacicus cela perteneciente a la familia Icteridae, dicha familia no fue analizada en el presente estudio.

De la misma manera se diferencia de un estudio realizado en 2019 en 4 puntos del eje de la carretera lquitos - Nauta [17] mediante el cual se determinó una prevalencia de malaria aviar de $83,1 \%$ en Cacicus cela.

Existen otros estudios en la zona tropical y sub tropical que determinaron la prevalencia de malaria aviar, utilizando una metodología de análisis de muestra distinta al de este estudio. Como el caso del estudio realizado por Herrera et al. [18] en el que utilizan métodos microscópicos para analizar una comunidad de aves, por el cual 271 muestras de sangre fueron analizadas determinando la prevalencia de malaria aviar en 19,05\%. Si bien el número de muestras analizadas es similar en número a nuestro estudio, la prevalencia fue mucho menor, este ontraste, en nuestra opinión no podría ser explicada por el ambiente, ya que ambos estudios fueron realizados en áreas dentro de zonas tropicales. La temperatura no sería un factor determinante para la proliferación de la infección en comunidades de aves. Sin embargo, podríamos inferir que el tipo de ensayo utilizado para el diagnóstico de la malaria, podria atribuirse a la gran diferencia observada. siendo el método molecular mucho más confiable y preciso, si arrojaría cifras mucho más reales acerca de la prevalencia en dichas comunidades.

Se han realizado otras investigaciones sobre prevalencia de malaria aviar en otro tipos de regiones climatológicas en nuestro país y en el continente. Por ejemplo, un estudio realizado en 2020 en la Reserva Nacional de Lachay (RNL) en la costa peruana sobre hemoparasitismo [19] determinaron prevalencia por hemosporidos de $24,82 \%$, resultado ligeramente superior al estudio realizado en la RNAM pero aún muy distante de la prevalencia del presente estudio

Finalmente el estudio realizado por Musa et al. [20] en 2019 en la selva lluviosa de Madagascar es el que muestras resultados más próximos a los nuestros, en este se estudió una comunidad de aves del cual se extrajo y analizo 1067 muestras sanguíneas por métodos moleculares, pudiendo determinar una prevalencia de $52,9 \%$ para el género plasmodium en 55 especies de aves pudiendo observarse resultados similares a los de este estudio.
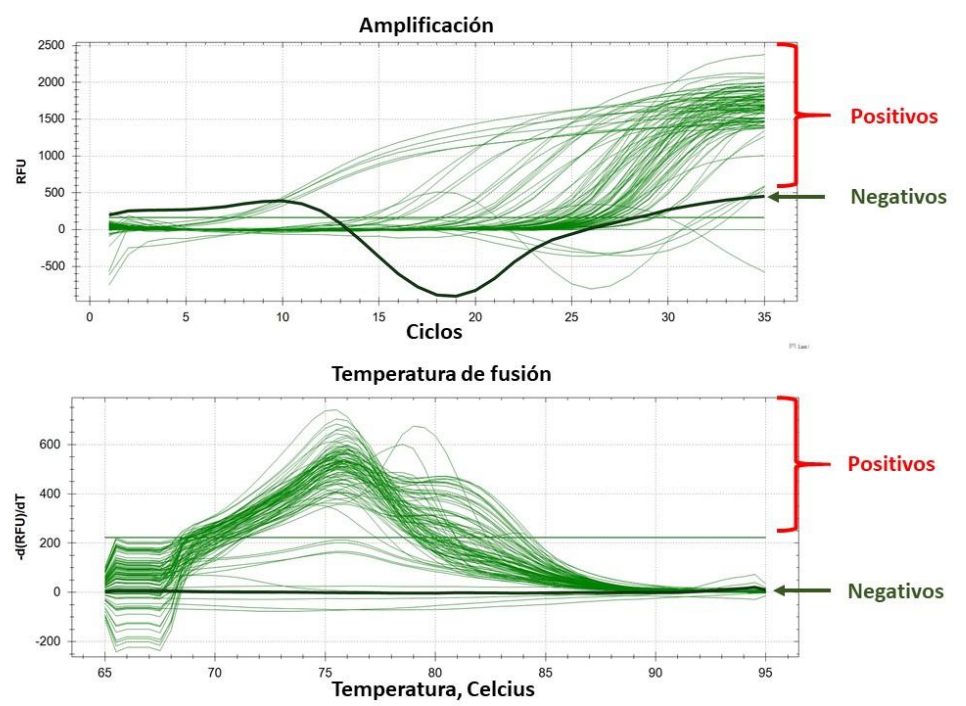

Figura 2: Detección de malaria aviar mediante reacción en cadena de la polimerasa en tiempo real 


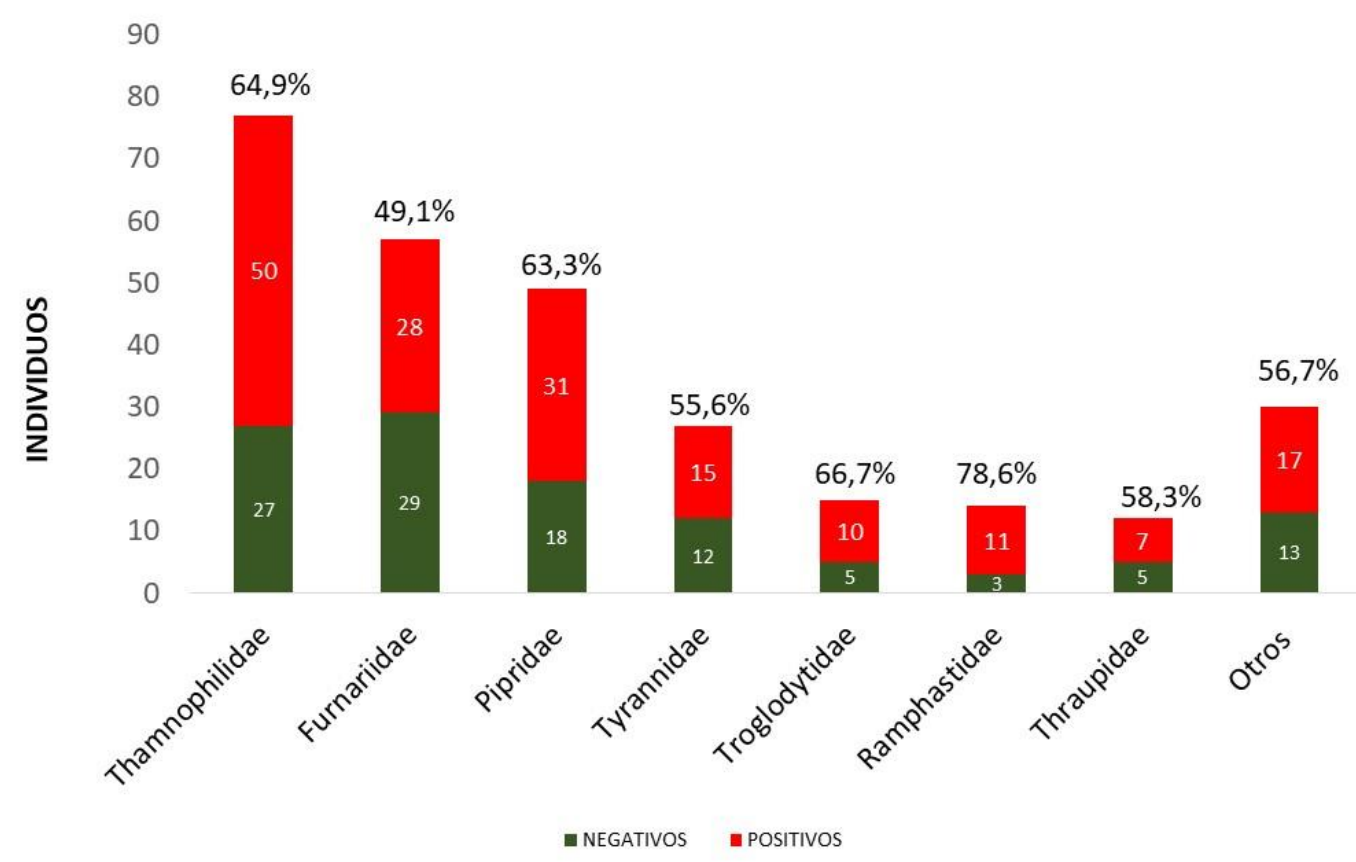

FAMILIA

Figura 3: Prevalencia de malaria aviar en la Cuenca Alta Río Itaya, Loreto, Perú

\section{Conclusiones}

La prevalencia de malaria en aves de sotobosque de la cuenca alta del rio Itaya es alta, pudiendo representar un riesgo para la conservación de la diversidad de la avifauna en la Amazonía peruana.

\section{Agradecimientos}

Al proyecto: "Desarrollo de un Biosensor portátil y versátil basado en el Sistema CRISPR/Cas para la detección rápida, sensible, específica y de bajo costo de múltiples patógenos que causan la malaria en aves en la Amazonía Peruana". Universidad Cientifica del Perú (UCP). FONDECYT/BM (Convenio 044-2019-FONDECYT/BM). por ser el ente financiero para realizar el presente trabajo de investigación.

\section{Referencias}

[1] D. Bahamonde, «Prevalencia de Malaria Aviar en las aves más comunes del Parque Recreacional-Bosque Protector Jerusalem, Pichincha, Ecuador», Pontificia Universidad Católica del Ecuador, Quito, 2014.

[2] C. T. Atkinson, R. J. Dusek, K. L. Woods, y W. M. Iko, «Pathogenicity of avian malaria in experimentally-infected Hawaii Amakihi», J. Wildl. Dis., vol. 36, n.o 2, pp. 197-201, abr. 2000, doi: 10.7589/0090-355836.2.197.

[3] M. Ilgūnas et al., «Patterns of Plasmodium homocircumflexum virulence in experimentally infected passerine birds", Malar. J., vol. 18, n.o 1, p. 174, dic. 2019, doi: 10.1186/s12936-019-2810-2

[4] L. Garcia-Longoria et al., "Diversity and host assemblage of avian haemosporidians in different terrestrial ecoregions of Peru», Curr. Zool., p. zoab030, abr. 2021, doi: 10.1093/cz/zoab030.

[5] N. J. Clark, S. Olsson-Pons, F. Ishtiaq, y S. M. Clegg, «Specialist enemies, generalist weapons and the potential spread of exotic pathogens: malaria parasites in a highly invasive bird», Int. J. Parasitol., vol. 45, n.o 14, pp. 891-899, dic. 2015, doi: 10.1016/j.jpara.2015.08.008.

[6] M. A. J. Moens y J. Pérez-Tris, «Discovering potential sources of emerging pathogens: South America is a reservoir of generalist avian blood parasites", Int. J. Parasitol., vol. 46, n.o 1, pp. 41-49, ene. 2016, doi: 10.1016/j.jpara.2015.08.001.

[7] G. Valkiūnas, «Haemosporidian vector research: marriage of molecular and microscopical approaches is essential: NEWS AND VIEWS: PERSPECTIVE», Mol. Ecol., vol. 20, n.o 15, pp. 3084-3086, ago. 
2011, doi:

294X.2011.05187.x.

Volumen 18, número 1 10.1111/j.1365-

[8] S. Merino et al., «Haematozoa in forest birds from southern Chile: Latitudinal gradients in prevalence and parasite lineage richness", Austral Ecol., vol. 33, n.o 3 , pp. 329-340, may 2008, doi: 10.1111/j.1442-9993.2008.01820.x.

[9] D. A. LaPointe, C. T. Atkinson, y M. D. Samuel, «Ecology and conservation biology of avian malaria: Ecology of avian malaria», Ann. N. Y. Acad. Sci., vol. 1249, n.o 1, pp. 211-226, feb. 2012, doi: 10.1111/j.17496632.2011.06431.x.

[10] K. S. S. Navas, «Diversidad y abundancia de especies de peces characiformes en tres quebradas de la concesión de conservación cuenca alta rio itaya, san juan bautista, loreto - perú», Universidad Científica del Perú.

[11] C. J. Ralph, G. R. Geupel, P. Pyle, T. E. Martin, D. F. DeSante, y B. Milá, «Manual de métodos de campo para el monitoreo de aves terrestres", U.S. Department of Agriculture, Forest Service, Pacific Southwest Research Station, Albany, CA, PSW-GTR-159, 1996. doi: 10.2737/PSWGTR-159.

[12] T. S. Schulenberg, Ed., Birds of Peru. Princeton: Princeton University Press, 2007.

[13] M. A. Plenge, «List of the Birds of Peru», p. 42.

[14] K. Rose, S. Newman, M. Uhart, y J. Lubroth, "Vigilancia de la influenza aviar altamente patógena en las aves silvestres», p. 58.

[15] P. Sunnucks y D. F. Hales, «Numerous transposed sequences of mitochondrial cytochrome oxidase I-II in aphids of the genus Sitobion (Hemiptera: Aphididae)», Mol. Biol. Evol., vol. 13, n.o 3, pp. 510-524, mar. 1996, doi: 10.1093/oxfordjournals.molbev.a025612.

[16] J. A. Bell, J. D. Weckstein, A. Fecchio, y V. V. Tkach, «A new real-time PCR protocol for detection of avian haemosporidians", Parasit. Vectors, vol. 8, n.o 1, p. 383, dic. 2015, doi: 10.1186/s13071-015-0993-0.

[17] J. Bell, «A Simple Way to Treat PCR Products Prior to Sequencing Using ExoSAP-IT», 6, vol. 44, p. 1, 2008.

[18] L. Ricopa y Z. Villa, «Prevalencia y diversidad de hemoparásitos en aves capturadas en la Reserva Nacional
Julio 2021

Allpahuayo Mishana (RNAM), Iquitos-Perú, 2013.pdf», Universidad Nacional de la Amazonía Peruana, 2016.

[19] L. Romero, «Prevalencia y análisis filogenético de hemoparásitos en colonias de cacicus cela "paucares" linnaeus, 1758 (passeriformes: icteridae).", Universidad Nacional de la Amazonía Peruana, lquitos Perú, 2019.

[20] J. Herrera, J. Pérez, Y. Fuentes, y C. Gantes, «Endoparasitos en aves residentes y migratorias de Punta Santa Bárbara, Parque nacional Darien, Panamá.pdf», Revista Colón Ciencias, Tecnología y Negocios, vol. 5. p. 19.

[21] R. Najarro, «Evaluación estacional de hemoparasitismo en las aves de la Reserva Nacional de Lachay.pdf», 2020.

[22] S. Musa, U. Mackenstedt, F. Woog, y A. Dinkel, «Avian malaria on Madagascar: prevalence, biodiversity and specialization of haemosporidian parasites", Int. J. Parasitol., vol. 49, n.o 3-4, pp. 199-210, mar. 2019, doi: 10.1016/j.jpara.2018.11.001.

E-mail:

paupamara271297@gmail.com

tavt89@gmail.com

sestelam@ucp.edu.pe

hiclernrm177unap@unapiquitos.edu.pe

juan.castro@unapiquitos.edu.pe

mcobos@ucp.edu.pe

jaedianap@gmail.com

idylanmaddox@gmail.com 